\title{
$\mathbf{X}$
}

\section{STELLAR ASTRONOMY}




\title{
Objective-Prism Spectroscopy: Retrospective and Prospective
}

\author{
D. Jack MacConnell \\ Astronomy Programs, Computer Sciences Corp. Space Telescope \\ Science Inst.
}

\begin{abstract}
The combination of the excellent large-field imaging capabilities of Schmidt telescopes and of prisms covering the full aperture has made an impact on stellar, galactic, and extragalactic astronomy far out of proportion to the numbers of such telescopes. I discuss some of the milestones in the continuing development of this combination with a view to signposts of the future.
\end{abstract}

\section{Origins}

Bernhard Schmidt was born 115 years ago this month; 1879 was also the year of birth of Albert Einstein, Otto Hahn, and Max von Laue. Joseph Fraunhofer was born 92 years earlier and died 53 years before Schmidt was born - like Schmidt, dying rather young. Fraunhofer is credited with the first use of an objective prism on a telescope, about 1823 , as well as with improvements in telescope optics, mountings, a wide array of analytical instruments, and discovery of the solar absorption features that bear his name. In the period 1863-1868, Fr. Angelo Secchi, S.J. at the Specola Vaticana in Rome was regularly using an objective prism for visual studies of bright stars, examining over 4000 and classifying them into four principal types according to their absorption features. A few years later, seven years before Schmidt's birth, the wealthy physician and early pioneer in photography, Henry Draper, made the first photograph of a stellar spectrum, Vega, through a quartz prism. Draper died 10 years later when Schmidt was 3, and his wife donated his instruments and a small sum of money to the Harvard College Observatory so that Draper's work on spectra might continue. The director was E.C. Pickering who made excellent use of the funds to equip several telescopes with objective prisms and photographic plateholders and to begin an extensive survey of stellar spectra over both hemispheres to 9 th visual magnitude. The classification system was developed by Williamina Fleming, Antonia Maury, and Pickering's student, Annie J. Cannon. According to Russell (1944), in 1910 Mrs. Fleming was the first person to know of the existence of white dwarfs after seeing the spectrum of 40 Eri B on the Draper Memorial plates. Another major discovery from these early Harvard plates was of spectroscopic binaries when Pickering noticed the periodic doubling of lines in $\zeta \mathrm{U} \mathrm{Ma}$. The major work, the Henry Draper Catalogue, was published in nine volumes in the period 1918-1924 and contained temperature types for 225,300 stars - a monumental effort for the pre-digital computer era. The notes on individual stars have been a rich source of material on peculiar spectra, the spectra 
of variables, and on differing luminosities and have been exploited by many observers. In the present day, we are thankful to have Dr. Nancy Houk who is re-classifying all the HD stars on the Morgan-Keenan system using excellent plates taken with the Curtis and Burrell Schmidt telescopes.

By 1930, Schmidt had developed the prototype of the telescope that bears his name at a unheard-of focal ratio of $1: 1.75$ and imaging a $15^{\circ}$ field completely coma-free. There must have been a pent-up need for such an instrument because, by 1941, there were two dozen Schmidt telescopes with primary diameter of $25 \mathrm{~cm}$ or larger! The largest one operating in the world at the end of 1941, until it was surpassed by the Tonantzintla Schmidt a few months later, was the 61/91-cm, f/3.5 Burrell telescope of Case Western Reserve Univ. (then called the Case School of Applied Science) equipped originally with a $4^{\circ}$ prism - the telescope our host, Dr. Hidayat, Dr. Houk, and I, among many others, used for our dissertations three decades ago. Its first and primary user was Prof. J.J. Nassau who pioneered in the use of near-infrared emulsions with the prism in the late 1940's. Nassau and Dr. Bruno van Albada, who later came to the Bosscha Observatory and was instrumental in obtaining funding for the "Bima Sakti" Schmidt, developed the classification scheme for the $M$ stars, and they and other co-workers conducted numerous studies of the distribution of cool giants over the northern Milky Way. The methods are summarized in Nassau (1956).

\section{Some earlier summaries of objective-prism work}

For the student wishing to review what has gone before, there are excellent summaries written for previous Schmidt conferences, and I recommend the articles by Stephenson (1966), Bidelman (1966, 1972), and McCarthy (1984). Also, in the triennial IAU Reports on Astronomy for Commission 45, there is a section on stellar classification from slitless and objective-prism spectra. There are also a number of articles of interest in Objective-Prism and Other Surveys (OPOS) edited by Philip and Upgren (1991).

\section{Some developments of the past decade}

Schmidt telescopes, with their excellent wide-field imaging capabilities, combined with prisms covering the full aperture, fine-grained plates, and a site with good seeing, provide a formidable tool for probing the structure and content of the Galaxy and members of the Local Group and for searching for distant emission-line and very blue galaxies. Much of the work done with this tool in the past decade has been devoted to surveys for QSOs (see, e.g. the conference proceedings edited by Osmer et al. 1988) which I will not cover except to say that there are several sources of "contamination" in such searches, e.g. faint, blue galactic stars such as some white dwarfs and subdwarfs, which are cast aside by extragalactic researchers but prized by others of us. The problem is that one often does not know which objects are stellar and which are not, until after considerable large-telescope time has been expended; in this regard, see the recent review paper of Hewett and Foltz (1994). In this review, I will confine myself largely to stellar work in our Galaxy done with objective prisms on Schmidt telescopes. 


\section{The dwarf carbon stars}

Having said that, I will now say a few words about a QSO-blue galaxy survey I have been associated with recently! When Nicholas Sanduleak died unexpectedly in May 1990 (within a few month's of Schmidt's age at death), Peter Pesch asked if I might help him continue the Case Low-Dispersion Northern Sky Survey. I agreed, and Bruce Stephenson also volunteered to help. Pesch and Sanduleak had reviewed their work at the Asiago Schmidt conference ten years ago when two papers in the series had been published, and at the time of Nick's death, ten more had appeared or were in press. In Paper XII of the series (Pesch et al. 1991), a summary is given of the areas covered and categories of objects published to that time. The papers present accurate coordinates and finding charts for a wide variety of objects, most marked for their strong blue continuum shortward of the Balmer limit down to $\sim 3300 \AA$. Extensive cross-referencing is also done which is a major effort. Objects marked as interesting are placed into eight categories with the first two reserved for resolved galaxies and unresolved blue and/or emission-line objects - the latter being QSO candidates of which over 1100 have been published. The remaining categories contain various types of halo objects expected or known to be stars such as white dwarfs, Pop II A-F stars, and F-G subdwarfs. Category VII, called 'CLS' (Case Late Stars), contains faint carbon and late $M$ suspected halo giants which seems an unusual group to be finding on plates taken for blue objects! Paper VII of the series appropriately contains the Category VII stars of which 26 are $\mathrm{C}$ or $\mathrm{C}$ : - followup spectroscopy by Bothun et al. (1991) and Green and Margon (1990) confirmed many of these as carbon stars. The interest in the faint $\mathrm{C}$ stars in the halo was that they should be at tens of $\mathrm{kpc}$ and therefore be useful as dynamic probes, so their radial velocities were measured. But this expectation was undercut by the finding that three CLS carbon stars, nos. 31, 50, and 96, have significant proper motions so cannot be distant (Green et al. 1991; 1992). They must then be members of the dwarf carbon class of which G77-61 was previously the lone member (discovered through its peculiar colours by Dahn et al. (1977)). This has many interesting implications for research on mass exchange in close binaries and on dynamic probes at large distances above the galactic plane, but they are outside the scope of my talk here.

It is interesting to note that two further dwarf carbon stars have been found from the spectrum of the companion white dwarf appearing on objective-prism plates, and the dwarf carbon was seen only after taking slit spectra redward of about $5000 \AA$. One of these was published as Case Blue Star 311 (Sanduleak \& Pesch 1989) and also as a uv-excess object in the Second Byurakan Survey (Stepanian et al. 1990), but Liebert et al. (1994) find the spectrum to be a composite DA/dC. A similar case is presented by PG $0824+289$ (Heber et al. 1993), not originally noticed on objective-prism plates, but found to be an Xray source in the ROSAT All-Sky Survey and then classified as a hot DA on an objective-prism plate taken with the Hamburg Schmidt at Calar Alto as part of the Hamburg-Munich collaboration to classify ROSAT-identified X-ray sources. This type conflicted with that given in the PG catalogue, so a slit spectrum was taken which revealed the presence of the dC star. As of early 1994, there were ten of these stars known, all expected to be members of close binaries where the degenerate star has lost its carbon-enriched photosphere to a lower-mass dwarf 
companion. They may be more common per unit volume than the classical giant carbon stars.

\section{The giant carbon stars}

Stephenson (priv. comm.) estimates that at least $70 \%$ of the $\mathrm{C}$ stars he has catalogued were found on objective-prism plates, and the percentage for S-type stars must be close to 80 . With such distinctive spectra, $C$ stars can be found rather easily in a variety of spectral regions and at quite low dispersion. Strictly speaking, $\mathrm{C}$ stars must have $C_{2}$ bands, but the presence of strong $\mathrm{CN}$ bands in the $\lambda \lambda 7900-8300 \AA$ region is sufficient to call them carbon on near-infrared plates. Westerlund continues to survey for faint $\mathrm{C}$ stars using plates taken with the Kvistaberg and ESO Schmidts (Ann. Rep. of Uppsala Obs., 1992), Kurtanidze and Nikolashvili at Abastumani have published large numbers of new, northern $\mathrm{C}$ stars, and Maehara and Soyano used the $4^{\circ}$ prism at the Kiso Schmidt to find several dozen new ones in selected longitude sectors. Fuenmayor continues searching for very faint $\mathrm{C}$ stars in selected regions of the galactic plane, and stars in his thesis (Fuenmayor 1981) have been used by Aaronson et al. (1990) and Metzger and Schechter (1994) to measure galactic rotation at large galactocentric distance. His star FJF 270 is one of a small number of C stars showing oxygen-rich silicates in the IRAS LRS spectrometer data (Lloyd Evans 1990, Little-Marenin et al. 1994).

\section{Luminous stars in the galactic plane}

There have been many surveys for luminous stars using Schmidt plates, and I will mention only one from recent years. Wiramihardja and Kogure (1987) used the Kiso Schmidt to find 1800 late B/early A stars and 128 emission-line objects in a field in C Ma. This is part of the long-standing and very successful cooperative program between our Indonesian hosts and Japanese astronomers. With such a great plethora of interesting objects on any objective-prism plate taken in the galactic plane, it is understandable that there is a long lag between the publication of a catalogue and subsequent follow-up observations. It certainly is true that the large majority of luminous stars of all types have been found on Schmidt objective-prism plates. We still see new observations published for stars listed in the Luminous Stars series of the Case and Hamburg Observatories (see Sanduleak and Stephenson 1971 and references therein), e.g. Drilling (1991), Reed (1993a; 1993b). In the last-cited paper, Reed used his photometry to show that some of the southern $L S$ stars are at distances greater than $5 \mathrm{kpc}$ and states that $31 \%$ of the southern $L S$ stars still have no photometry. On the other hand, not all of the stars published in the Luminous Stars series have turned out to be luminous. Drilling (e.g. 1987) has found many in both hemispheres which turn out to be hot and subluminous or degenerate, and Parthasarathy (1993) has recently argued that $L S+34^{\circ} 26$ is not a massive Pop I star but is a low-mass, post-AGB B1.5 supergiant with a detached, cold, circumstellar dust shell causing it to be an IRAS source.

\section{Surveys for emission-line objects}

A wide variety of emission-line objects are found on objective-prism plates, e.g. Be stars, Wolf-Rayets, some supergiants, Miras, T Tau and T Tau-like stars, 
dMe stars, H II regions, planetary nebulae, QSOs, and active galaxies. There is probably not an observatory with a Schmidt and prism that has not published at least one list of emission-line objects! I won't attempt to summarize the activity in the past decade but will draw attention to the work of Briceño et al. (1993 and these proceedings). They have used the 1-m Schmidt at CIDA in Venezuela to survey the Tau-Aur region for faint $\mathrm{H} \alpha$-emission stars down to $V$ $\sim 18$ over 50 sq. deg. and found 12 new stars which were confirmed as pre-main sequence on big-telescope slit spectra. They are all later than about M0 and have masses down to about 0.1 solar. These are the first results of their survey and among the first results from that telescope, and I hope we will be seeing many more contributions from them and the others in Venezuela. Wiramihardja et al. (1993) have also been active in recent years in surveying for $\mathrm{H} \alpha$-emission stars in the Orion region using plates taken with the Kiso and Curtis Schmidts.

\section{Searches out of the plane}

There have been a number of important papers in recent years using objectiveprism plates to search for metal-weak stars along the lines of the seminal work of Bond $(1970,1980)$. This method avoids the obvious kinematical bias introduced in using proper motion samples to study the space density and luminosity function of halo stars. Beers et al. $(1985,1988,1992)$ used the $4^{\circ}$ prism on the Curtis Schmidt together with an intermediate-band interference filter centered between the $\mathrm{Ca}$ II $\mathrm{H}$ and $\mathrm{K}$ lines to search for very metal-weak stars to $B \sim 16$. They searched for stars with weak or absent $\mathrm{Ca}$ II and found several thousand blue field horizontal-branch stars and a large mix of other peculiar stars including some of the most metal-deficient stars known to date. Flynn and Morrison (1990) and Morrison et al. (1990) carried out a survey in three directions using the full spectral region of $\lambda \lambda 3500-5300 \AA$ with AAO and Uppsala Schmidt plates to semi-automatically segregate metal-weak $\mathrm{G}$ and $\mathrm{K}$ giants. They criticized the Beers et al. work for selection effects and sampled the full range of abundances from disk to halo. Among their many conclusions on the kinematics of various abundance groups, they find stars in the abundance range $-1.6 \leq[\mathrm{Fe} / \mathrm{H}] \leq-1.0$ that have kinematical and spatial properties similar to the thick disk stars and refer to them as the "metal-weak thick disk" stars. More recently, Morrison (1993) used the objective-prism survey results of Bidelman and MacConnell (1973) to conclude that the local density of halo giants is a factor of two less than found by previous studies, a ratio of 1:850, halo-to-disk. In the following article in the A.J., Rodgers et al. (1993) present a catalogue of stars earlier than $F 5$ in two directions $45^{\circ}$ out of the plane using $600 \AA / \mathrm{mm}$ plates from the AAO Schmidt. In the area in common, they find good agreement with the list of Beers et al. (1988) and recover a number of known RR Lyraes, hot sub-dwarfs, and white dwarfs.

At a higher dispersion than the above surveys, $85 \AA / \mathrm{mm}$, Rose (1991) and Rose and Agostinho (1991) used the Curtis Schmidt isolating a 135- $\AA$ section of spectrum centered on $4080 \AA$ with the objective of determining the distribution in abundance, kinematics, and age of the galactic disk as a function of height above the galactic plane to a distance of $\sim 2 \mathrm{kpc}$. They take six plates of each field, then digitize and co-add, and their survey reaches to $B \sim 13$. They find they can distinguish 7 levels of resolution between giants and dwarfs down to 
$B \leq 12$ for $\mathrm{F}-\mathrm{K}$ stars and 3 to 4 levels down to the plate limit. For $B>11$, there is a fairly sharp turn-on in the F-star distribution at F7. With their collaborator J. Stock, they are also obtaining radial velocities by reversing the prism orientation between sets of plates. This method has had extensive development and testing by Stock and is discussed by him in $O P O S$ and in Stock (1992) where he gives a table of 1300 stars in two regions. Thus, from one set of plates, these workers obtain not only surface gravity and abundance information but also radial velocities and have the possibility of calculating proper motions using their accurate coordinates and those of earlier positional catalogues. I want to close this section by mentioning the objective-prism work of two researchers who ordinarily confine their work to slit spectra, Chris Corbally and Bob Garrison (Corbally and Garrison 1988a, 1988b). They have found about 200 early $G$ dwarfs in a 4 sq. deg. region at each galactic pole down to $V \sim$ 15.7 on thin-prism plates from the Curtis and Burrell Schmidts. These stars are good probes of the halo structure because they are homogeneous and show little effect of photospheric mixing unlike the more evolved stars, and they are the brightest of the unevolved stars with ages approaching that of the halo.

\section{Prospective}

The future is longer than the past, but the proportion will not be reflected in this review! We are well into the digital era in astronomy, and this profoundly affects everything we do. Plates taken years ago are now being scanned by rapid machines transforming grains into bytes which can then be co-added, manipulated in a variety of ways, and shipped around the world in a moment. The digitization of spectrum plates is being done by several groups, and we can expect that trend to grow in the future. We hope to see automated classification of large numbers of non-HD stars on the plates Nancy Houk is using and perhaps improved classification of the brighter stars as well. But the quality of the original plates is fundamental to the process, and we must take care to preserve and protect them even after they have been "turned into pixels". Some groups which have been very active in photographic spectrum survey work for many years now find it difficult to come up with new ideas for meaningful surveys unless large-format CCDs are employed. Even then, the pixel size will have to be smaller than current ones to fully take advantage of the best seeing at the best sites, and the detectors will have to have correspondingly more pixels to cover a decent piece of sky, say, at least $1^{\circ}$ on a side. Currently, with $15 \mu$ pixels, a $2048 \times 2048$ detector on the Burrell or Curtis Schmidt will cover about $50^{\prime}$ on a side at a pixel size of 1.45 . To take advantage of $0 ! 75$ seeing and using the $10^{\circ}$ prism on either of these telescopes, one needs $8 \mu$ pixels and 2500 of them to cover the spectrum to $5000 \AA$. To cover a one-degree square, one needs a 4800 $x 4800$ chip which would be about the length of two such spectra. Since there is no need to widen the spectra, one can expect to reach $B \sim 18$ in an 8-min exposure.

Pesch (priv. comm.) states that most of the work done so far with the CCD on the Burrell telescope at Kitt Peak has been in the direct mode, and that, in the most-recent semester, it was the most-sought telescope on the mountain. The problem with CCD objective-prism work, he points out, is the sheer amount 
of data handling involved, and nobody has thus far picked out all the spectra, gotten their positions, scanned, and classified them; some parts of the task have been done and tested but not the full ensemble. However, it is a matter of time and probably a fair amount of money before this too is accomplished, and we will have a clearer view than ever before of the nature of our Galaxy and universe and of the marvelous images produced by Schmidt telescopes.

\section{References}

Aaronson M., Blanco V.M., Cook K.H., Olszewski E.W. \& Schechter P.L., 1990, ApJS, 73, 841

Beers T.C., Preston G.W. \& Schectman S.A., 1985, AJ, 90, 2089

Beers T.C., Preston G.W. \& Schectman S.A., 1988, ApJS, 67, 461

Beers T.C., Preston G.W. \& Schectman S.A., 1992, AJ, 103, 1987

Bidelman W.P., 1966, Vistas in Astr., 8, 53

Bidelman W.P., 1972, in Proceedings of the Conference on The Role of Schmidt Telescopes in Astronomy, U. Haug, ed., (Hamburger Sternwarte), p. 53

Bidelman W.P. \& MacConnell D.J., 1973, AJ, 78, 687

Bond H.E., 1970, ApJS, 22, 117

Bond H.E., 1980, ApJS, 44, 517

Bothun G., Elias J.H., MacAlpine G., Matthews K., Mould J.R., Neugebaner G. \& Reid I.N., 1991, AJ, 101, 2220

Briceño C., Calvet N., Gomez M., Hartmann L.W., Kenyon S.J. \& Whitney B.A., 1993, PASP, 105, 686

Corbally C.J., S.J. \& Garrison R.F., 1988a, AJ, 95, 739

Corbally C.J., S.J. \& Garrison R.F., 1988b, AJ, 95, 745

Dahn C.C., Liebert J., Kron R.G., Spinrad H. \& Hintzen P.M., 1977, ApJ, 216, 757

Drilling J.S., 1987, in The Second Conference on Faint Blue Stars, Proc. IAU Coll. 95, A.G.D. Philip, D.S. Hayes \& J.W. Liebert, eds, (Davis, Schenectady NY), p. 489

Drilling J.S., 1991, ApJS, 76, 1033

Flynn C. \& Morrison H.L., 1990, AJ, 100, 1181

Fuenmayor F.J., 1981, Rev. Mex. Astr. Ap., 6, 83

Green P.J. \& Margon B., 1990, PASP, 102, 1372

Green P.J., Margon B. \& MacConnell D.J., 1991, ApJ, 380, L31

Green P.J., Margon B. \& MacConnell D.J., 1992, ApJ, 400, 659

Heber U., Bade N., Jordan S. \& Voges W., 1993, A\&A, 267, L31

Hewett P.C. \& Foltz C.B., 1994, PASP, 106, 113

Liebert J., Schmidt G.D., Lesser M., Stepanian J.A., Lipovetsky V.A., Chaffee F.H., Foltz C.B. \& Bergeron P., 1994, ApJ, 421, 733

Little-Marenin I.R., Sahai R., Wannier P.G., Benson P.J., Gaylard M. \& Omont A., 1994, A\&A, 281, 451 
Lloyd Evans T., 1990, MNRAS, 243, 336

McCarthy M.F., S.J., 1984, in Astronomy with Schmidt-Type Telescopes, Proc.

IAU Coll. 78, M. Capacciolo, ed., p. 37

Metzger M.R. \& Schechter P.L., 1994, ApJ, 420, 177

Morrison H.L., Flynn C. \& Freeman K.C., 1990, AJ, 100, 1191

Morrison H.L., 1993, AJ, 106, 578

Nassau J.J., 1956, Vistas in Astr., 2, 1361

Osmer P.S., Porter A.C., Green R.F. \& Foltz C.B., eds., 1988, Proceedings of a Workshop on Optical Surveys for Quasars, ASP Conf. Ser. 2

Parthasarathy M., 1993, ApJ, 414, L109

Pesch P., Sanduleak N. \& Stephenson C.B., 1991, ApJS, 76, 1043

Philip A.G.D. \& Upgren A.R., eds., 1991, Objective-Prism and Other Surveys, (Davis, Schenectady NY)

Reed B.C., 1993a, AJ, 106, 2291

Reed B.C., 1993b, PASP, 105, 1465

Rodgers A.W., Roberts W.H. \& Walker I., 1993, AJ, 106, 591

Rose J.A., 1991, AJ, 101, 937

Rose J.A. \& Agostinho R., 1991, AJ, 101, 950

Russell H.N., 1944, AJ, 51, 13

Sanduleak N. \& Pesch P., 1989, ApJS, 70, 173

Stepanian J.A., Lipovetsky V.A. \& Eratsova L.K., 1990, Astrophyzica, 32, 441

Stephenson C.B., 1966, Vistas in Astr., 7, 59

Stephenson C.B. \& Sanduleak N., 1971, Pub. Warner \& Swasey Obs., 1, No. 1 Stock J., 1992, Rev. Mex. Astr. Ap., 24, 45

Wiramihardja S.D. \& Kogure T., 1987, Contrib. Bosscha Obs., No. 96 Wiramihardja S.D., Kogure T., Yoshida S., Ogura K. \& Nakano M., 1993, PASJ, 45,643 